\title{
Post-entry events of efficient R5 vs. inefficient X4 HIV-I replication in primary CD4+T lymphocytes, a transcriptome analysis Samanta A Mariani ${ }^{1}$, Andrea Crotti ${ }^{1}$, Elisa Vicenzi ${ }^{1}$, Huang Dawei ${ }^{2}$, Richard Lempicky², Claudia Cicala ${ }^{3}$, James Arthos ${ }^{3}$ and Guido Poli*1
}

Address: ${ }^{1}$ Department of Immunology, Transplantation and Infectious Diseases, San Raffaele Scientific Institute, Milano 20132, Italy, ${ }^{2}$ Science Applications International Corporation, Frederick, MD 21702, USA and ${ }^{3}$ National Institute of Allergy and Infectious Diseases, Bethesda, MD 20892, USA

* Corresponding author

from Frontiers of Retrovirology: Complex retroviruses, retroelements and their hosts Montpellier, France. 21-23 September 2009

Published: 24 September 2009

Retrovirology 2009, 6(Suppl 2):II9 doi:10.1186/I742-4690-6-S2-119

This abstract is available from: http://www.retrovirology.com/content/6/S2/II 9

(c) 2009 Mariani et al; licensee BioMed Central Ltd.

HIV-1 infects CD4+ cells via interaction with CD4 and either CCR5 or CXCR4. However, only CCR5-using (R5) viruses are efficiently transmitted and sustain the viral pandemics, while CXCR4-using (X4) viruses emerge later in coincidence with the immunodeficiency state and progression to AIDS in about $50 \%$ of individuals infected with subtype B HIV-1, but not with other subtypes. Unraveling cellular and molecular correlates of this asymmetric co-receptor use would be relevant to understand HIV pathogenesis as well as for the development of preventive strategies aimed at blocking R5 HIV-1 spreading. We have previously reported that cord blood derived CD4+T cells (CB4 cells) maintained in a sub-optimally activated state in IL-2 enriched medium for 7-14 days before infection are permissive for R 5 and restricted for X4 HIV-1 replication. Of interest, this restriction did not occur at the level of viral entry, but it was rather correlated to a superior capacity of R5 HIV-1 to spread after infection [1]. In the present study, we examined the transcriptomic profile at different time points $(8,24,48,72 \mathrm{~h})$ of CB4 cells established from 6 independent donor/infection pairs after infection with isogenic NL4-3 (X4) and NLAD8 (R5) viruses normalized for MOI. Gene expression was measured using Human Genome U95A chips and analyzed with the DAVID knowledge base software. Approximately 900 and 1,100 genes were selectively mobilized by R5 and X4 HIV-1 infection, respectively, vs. mock-stimulated uninfected control cells. An additional 420 genes were modulated by both viruses vs. controls. R5
HIV-1 induced a rapid mobilization of genes linked to cell proliferation and signal transduction, whereas the X4 virus predominantly modulated the expression of genes associated with cell death and the immune response. Both viruses upregulated the expression of CXCL12/SDF-1 $\alpha$, but only X4 downregulated CXCR4 mRNA; CCR 5 mRNA was unaffected by either infection at all time points. Other genes previously linked to control of HIV replication that were modulated by $\mathrm{R} 5$ and X4 HIV-1 include APOBEC3G, IFN- $\gamma$, CCL5/RANTES, CCL7/MCP-3 and CCL14/ HCC1. We are currently analyzing additional genes discordantly co-modulated by $\mathrm{R} 5$ and $\mathrm{X} 4$ viruses in the search of host genes associated with the permissive vs. restricted HIV replicative profile in this model system. Thus, both R5 and X4 HIV-1 profoundly affect the transcriptional activity of primary CD4+T lymphocytes even in the absence of overt replication (as observed in $\mathrm{X} 4$ infection).

\section{References}

I. Vicenzi E, Bordignon PP, Biswas P, Brambilla A, Bovolenta C, Cota M, Sinigaglia F, Poli G: Envelope-dependent restriction of human immunodeficiency virus type I spreading in CD4(+) T lymphocytes: $R 5$ but not $X 4$ viruses replicate in the absence of T-cell receptor restimulation. J Virol 1999, 73:7515-7523. 